\title{
Inverse Transformation of Elliptical Relative State Transition Matrix
}

\author{
Jianfeng Yin ${ }^{*}$, Yinrui Rao², Chao Han² \\ ${ }^{1}$ Institute of Spacecraft System Engineering, CAST, Beijing, China \\ ${ }^{2}$ School of Astronautics, Beihang University, Beijing, China \\ Email: ‘ianfengabc@gmail.com
}

Received 30 May 2014; revised 28 June 2014; accepted 25 July 2014

Copyright (C) 2014 by authors and Scientific Research Publishing Inc.

This work is licensed under the Creative Commons Attribution International License (CC BY).

http://creativecommons.org/licenses/by/4.0/

(c) (i) 0pen Access

\begin{abstract}
A new set of relative orbit elements (ROEs) is used to derive a new elliptical formation flying model in previous work. In-plane and out-of-plane relative motions can be completely decoupled, which benefits elliptical formation design. In order to study the elliptical control strategy and perturbation effects, it is necessary to derive the inverse transformation of the relative state transition matrix based on relative orbit elements. Poisson bracket theory is used to obtain the linear transformations between the two representations: the relative orbit elements and the geocentric orbital frame. In this paper, the details of these transformations are presented.
\end{abstract}

\section{Keywords}

Relative Orbit Elements, Elliptical Formation Flying, Relative State Transition Matrix, Inverse Transformation, Poisson Bracket

\section{Introduction}

In previous papers, an alternative method to study the dynamics of elliptical formation flying based on a new set of relative orbit elements was presented. The new relative orbit elements are very useful to obtain the geometric characteristics of relative motion. The principle of the method is simple. To make the ROE-based method more understandable, it is necessary to derive the transformations between the two representations: the relative orbit elements and the geocentric orbital frame.

Historically, the first article that presented these transformations was [1], but the author did not use them for relative motion. Other transformations were used by Garrison et al. [2] to obtain the equations of the relative motion for an elliptical reference orbit. Alfriend proposed another way to obtain the transformations and intro-

*Corresponding author. 
duced another set of orbital elements in Reference [3]. Using Alfriend's method, also called geometric method, Gim [4] introduced J2 perturbations and Sengupta introduced second order effects [5]. This solution has none of the drawbacks of Hill and Lawden equations. It can take into account second order effects, eccentric reference orbit, and perturbations. This solution is very well-adapted for control and navigation. Fontdecaba [6] used the Poisson brackets [7] to have transformations between the two representations: the difference of orbital elements and the local orbital frame.

Using the spherical geometry, Han's relative orbit elements are strictly defined through the employment of a projection rule [8]. A new elliptical relative motion model with no singularity problem based on the relative orbit elements is derived [9] [10]. Although the relative orbit elements are derived very differently from the differences of orbital elements, the transformation between the two methods can also be obtained. In order to analyze the perturbation effects and the control strategy, the inverse transformation of relative state transition matrix is greatly needed. Poisson brackets method is used to derive the inverse transformation in this paper.

The structure of the paper is as follows. The first section is the introduction. The second section will briefly introduce the new set of relative orbit elements, which are relative average drift rate, relative eccentricity vector, relative inclination vector, and difference of mean argument of latitude. The transformation between the relative orbit elements and the difference of orbital elements is given. Then the elliptical relative motion model based on the improved ROE theory is derived. The inverse transformation of the relative state transition matrix is obtained to analyze the perturbation effects and control strategy in the third section. The end is the conclusion.

\section{Relative Motion Equations}

\subsection{Related Coordinate Systems}

1) $\boldsymbol{F}_{o}$ is spacecraft-centered local vertical local horizontal coordinate system, with $z$-axis pointing to Earth's center, $x$-axis perpendicular to $z$-axis in the direction of the instantaneous velocity and $y$-axis perpendicular to the orbit plane.

2) $\boldsymbol{F}_{n}$ is Earth-centered node coordinate system, with $x$-axis pointing toward ascending node, $z$-axis in the direction of the moment of orbit motion, and $y$-axis completing the right-hand rule in the orbit plane.

3) $\boldsymbol{F}_{o}$ is geocentric orbital coordinate system, with $x$-axis pointing to spacecraft, $z$-axis in the direction of the moment of orbit motion, and $y$-axis completing the right-hand rule in the orbit plane.

4) $\boldsymbol{F}_{I}$ is Earth-centered inertial coordinate system, with $x$-axis pointing to the vernal equinox, $z$-axis fixed at celestial pole, and $y$-axis completing the right-hand rule in the equatorial plane.

\subsection{Definition of Relative Orbit Elements}

As introduced in the classical Keplerian orbits, the movement of a satellite is mathematically described by six quantities, called orbital elements, which are semi-major axis, $a$, eccentricity, $e$, inclination, $i$, right ascension of ascending node, $\Omega$, argument of perigee, $\omega$, and mean anomaly, $M$. For the relative motion between two close satellites, which means $\Delta r / a_{0} \ll 1, \Delta r$ is the relative distance, some simplifications can be made. Through first-order linear expansion, the relative orbit elements can be expressed as [8]

$$
\begin{aligned}
& \Delta e_{x}=e_{1} \cos \omega_{1}-e_{0} \cos \omega_{0}-e_{1} \sin \omega_{1} \Delta \Omega \cos i_{0} \\
& \Delta e_{y}=e_{1} \sin \omega_{1}-e_{0} \sin \omega_{0}+e_{1} \cos \omega_{1} \Delta \Omega \cos i_{0} \\
& \Delta i_{x}=\left(\Omega_{1}-\Omega_{0}\right) \sin i_{1} \\
& \Delta i_{y}=i_{0}-i_{1} \\
& \Delta M^{\prime}=\left(\omega_{1}-\omega_{0}\right)+\left(M_{1}-M_{0}\right)+\left(\Omega_{1}-\Omega_{0}\right) \cos i_{0}
\end{aligned}
$$

where $n_{*}$ is the mean motion of the satellite, $n_{*}=\sqrt{\mu / a_{*}^{3}} \cdot \mu$ is gravitational coefficient. In this paper, the quantities referred to the chief satellite are denoted by $(\cdot)_{0}$, and those related to the deputy satellite by $(\cdot)_{1}$. The subscript * means the subscript 0 or 1 . These 6 parameters in Equation (1) can be described as relative average drift rate $D$, relative eccentricity vector $\Delta \boldsymbol{e}=\left(\Delta e_{x}, \Delta e_{y}\right)$, relative inclination vector $\Delta \boldsymbol{i}=\left(\Delta i_{x}, \Delta i_{y}\right)$, and difference of mean argument of latitude $\Delta M^{\prime}$. They are also called the relative orbit elements of the deputy satellite with respect to the chief satellite.

Equation (1) can be written as following 


$$
\left[\begin{array}{c}
D \\
\Delta e_{x} \\
\Delta e_{y} \\
\Delta i_{x} \\
\Delta i_{y} \\
\Delta M^{\prime}
\end{array}\right]=\left[\begin{array}{cccccc}
-\frac{3 n}{2 a} & 0 & 0 & 0 & 0 & 0 \\
0 & \cos \omega & 0 & -e \operatorname{cosisin} \omega & -e \sin \omega & 0 \\
0 & \sin \omega & 0 & e \operatorname{cosicos} \omega & e \cos \omega & 0 \\
0 & 0 & 0 & \sin i & 0 & 0 \\
0 & 0 & -1 & 0 & 0 & 0 \\
0 & 0 & 0 & \operatorname{cosi} & 1 & 1
\end{array}\right]\left[\begin{array}{c}
\Delta a \\
\Delta e \\
\Delta i \\
\Delta \Omega \\
\Delta \omega \\
\Delta M
\end{array}\right]
$$

The orbital elements in Equation (2) are all referred to the orbital elements of the chief satellite. In the remainder of the paper, the subscript 0 will be omitted unless declared. Let

$$
\mathbf{R O E}=\left[\begin{array}{llllll}
D & \Delta e_{x} & \Delta e_{y} & \Delta i_{x} & \Delta i_{y} & \Delta M^{\prime}
\end{array}\right]^{\mathrm{T}}, \quad \Delta \mathbf{O E}=\left[\begin{array}{llllll}
\Delta a & \Delta e & \Delta i & \Delta \Omega & \Delta \omega & \Delta M
\end{array}\right]^{\mathrm{T}}, \quad \boldsymbol{\Phi}=\frac{\partial \mathbf{R O} \mathbf{E}^{\mathrm{T}}}{\partial \Delta \mathbf{O E}^{\mathrm{T}}}
$$

then Equation (2) can be written as

$$
\mathbf{R O E}=\boldsymbol{\Phi} \Delta \mathbf{O E}
$$

Seen from Equation (3), the transformation between the relative orbit elements and the difference of orbital elements can be obtained.

\subsection{Relative Motion Equations}

Seen from Figure 1, using the method of coordinate transfer and one-order linear expansion, the linear ROEbased relative motion equations expressed in the $\boldsymbol{F}_{o}$ frame of the chief satellite can be easily derived as

$$
\left\{\begin{array}{l}
x=\left[1+\left(\frac{r}{a}\right) \frac{1}{1-e^{2}}\right] a\left(\Delta e_{x} \sin u-\Delta e_{y} \cos u\right)+\sqrt{1-e^{2}}\left(\frac{a}{r}\right) a \Delta M^{\prime}(t)+\Delta_{1} \\
y=\left(\frac{r}{a}\right) a\left(\Delta i_{x} \cos u+\Delta i_{y} \sin u\right) \\
z=a\left(\Delta e_{x} \cos u+\Delta e_{y} \sin u\right)+\left(\frac{r}{a}\right) a \frac{2 D}{3 n}+\Delta_{2}
\end{array}\right.
$$

where

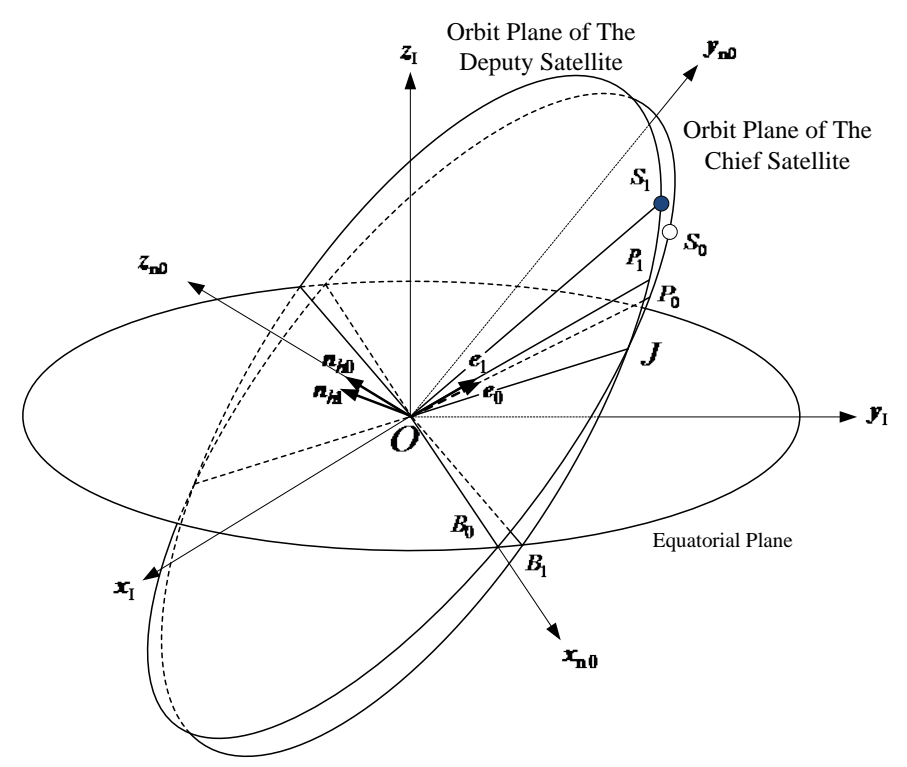

Figure 1. Relative motion relationship of two satellites. 


$$
\begin{gathered}
\Delta_{1}=\left(\frac{r}{a}\right)\left[\left(\frac{1-\sqrt{1-e^{2}}}{e}-e\right)\left(\frac{a}{r}\right)^{2}-\frac{e}{1-e^{2}}\right] a\left(\Delta e_{y} \cos \omega-\Delta e_{x} \sin \omega\right) \\
\Delta_{2}=-\frac{a \sin \theta}{\sqrt{1-e^{2}}}\left(e \Delta M^{\prime}(t)+\left(\sqrt{1-e^{2}}-1\right)\left(\Delta e_{y} \cos \omega-\Delta e_{x} \sin \omega\right)\right)
\end{gathered}
$$

where $\Delta M^{\prime}(t)=\Delta M^{\prime}\left(t_{0}\right)+D\left(t-t_{0}\right), t_{0}$ is the epoch time. $\boldsymbol{r}$ is the position vector in $\boldsymbol{F}_{I}$ frame and $r=\|\boldsymbol{r}\|$.

Using the first derivative of Equation (4) with respect to time $t$, the velocity equations of elliptical relative motion can be expressed as

$$
\left\{\begin{aligned}
v_{x}= & a n \sqrt{1-e^{2}}\left(\frac{a}{r}\right)^{2}\left[1+\left(\frac{r}{a}\right) \frac{1}{1-e^{2}}\right]\left(\Delta e_{x} \cos u+\Delta e_{y} \sin u\right)+\frac{a n}{1-e^{2}} \frac{e \sin \theta}{\sqrt{1-e^{2}}}\left(\Delta e_{x} \sin u-\Delta e_{y} \cos u\right) \\
& -a n e \sin \theta\left(\frac{a}{r}\right)^{2} \Delta M^{\prime}(t)+a \sqrt{1-e^{2}}\left(\frac{a}{r}\right) D+\frac{\mathrm{d} \Delta_{1}}{\mathrm{~d} t} \\
v_{y}= & a n \sqrt{1-e^{2}}\left(\frac{a}{r}\right)\left(-\Delta i_{x} \sin u+\Delta i_{y} \cos u\right)+a n \frac{e \sin \theta}{\sqrt{1-e^{2}}}\left(\Delta i_{x} \cos u+\Delta i_{y} \sin u\right) \\
v_{z}= & a n \sqrt{1-e^{2}}\left(\frac{a}{r}\right)^{2}\left(-\sin u \Delta e_{x}+\cos u \Delta e_{y}\right)+a n \frac{e \sin \theta}{\sqrt{1-e^{2}}} \frac{2 D}{3 n}+\frac{\mathrm{d} \Delta_{2}}{\mathrm{~d} t}
\end{aligned}\right.
$$

where $\frac{\mathrm{d} \Delta_{1}}{\mathrm{~d} t}=-a n \frac{e \sin \theta}{\sqrt{1-e^{2}}}\left[\left(\frac{1-\sqrt{1-e^{2}}}{e}-e\right)\left(\frac{a}{r}\right)^{2}+\frac{e}{1-e^{2}}\right]\left(\Delta e_{y} \cos \omega-\Delta e_{\chi} \sin \omega\right)$,

$\frac{\mathrm{d} \Delta_{2}}{\mathrm{~d} t}=-\frac{a e \sin \theta}{\sqrt{1-e^{2}}} D-\operatorname{anesin} \theta\left(\frac{a}{r}\right)^{2}\left(\Delta M^{\prime}(t)-\frac{1-\sqrt{1-e^{2}}}{e}\left(\Delta e_{y} \cos \omega-\Delta e_{x} \sin \omega\right)\right)$.

Equation (4) and Equation (5) are referred as the elliptical relative motion equations.

\section{The Inverse Transformation}

\subsection{Relative State Transition Matrix}

Equation (4) and Equation (5) can be rewritten as the form of state transition matrix as bellow

$$
\begin{aligned}
& \boldsymbol{X}(t)=\boldsymbol{M}(t)\left[\begin{array}{llllll}
D & \Delta e_{x} & \Delta e_{y} & \Delta i_{x} & \Delta i_{y} & \Delta M^{\prime}
\end{array}\right]^{\mathrm{T}} \\
& \boldsymbol{M}(t)=\left[\begin{array}{cccccc}
\frac{\partial x}{\partial D} & \frac{\partial x}{\partial \Delta e_{x}} & \frac{\partial x}{\partial \Delta e_{y}} & 0 & 0 & \frac{\partial x}{\partial \Delta M^{\prime}} \\
0 & 0 & 0 & \frac{\partial y}{\partial \Delta i_{x}} & \frac{\partial y}{\partial \Delta i_{y}} & 0 \\
\frac{\partial z}{\partial D} & \frac{\partial z}{\partial \Delta e_{x}} & \frac{\partial z}{\partial \Delta e_{y}} & 0 & 0 & \frac{\partial z}{\partial \Delta M^{\prime}} \\
\frac{\partial v_{x}}{\partial D} & \frac{\partial v_{x}}{\partial \Delta e_{x}} & \frac{\partial v_{x}}{\partial \Delta e_{y}} & 0 & 0 & \frac{\partial v_{x}}{\partial \Delta M^{\prime}} \\
\frac{0}{0} & 0 & \frac{\partial v_{y}}{\partial \Delta i_{x}} & \frac{\partial v_{y}}{\partial \Delta i_{y}} & 0 \\
\frac{\partial v_{z}}{\partial D} & \frac{\partial v_{z}}{\partial \Delta e_{x}} & \frac{\partial v_{z}}{\partial \Delta e_{y}} & 0 & 0 & \frac{\partial v_{z}}{\partial \Delta M^{\prime}}
\end{array}\right]
\end{aligned}
$$

The inverse matrix $\boldsymbol{M}^{-1}$ can be expressed as 


$$
\boldsymbol{M}^{-1}=\left[\begin{array}{cccccc}
\frac{\partial D}{\partial x} & 0 & \frac{\partial D}{\partial z} & \frac{\partial D}{\partial v_{x}} & 0 & \frac{\partial D}{\partial v_{z}} \\
\frac{\partial \Delta e_{x}}{\partial x} & 0 & \frac{\partial \Delta e_{x}}{\partial z} & \frac{\partial \Delta e_{x}}{\partial v_{x}} & 0 & \frac{\partial \Delta e_{x}}{\partial v_{z}} \\
\frac{\partial \Delta e_{y}}{\partial x} & 0 & \frac{\partial \Delta e_{y}}{\partial z} & \frac{\partial \Delta e_{y}}{\partial v_{x}} & 0 & \frac{\partial \Delta e_{y}}{\partial v_{z}} \\
0 & \frac{\partial \Delta i_{x}}{\partial y} & 0 & 0 & \frac{\partial \Delta i_{x}}{\partial v_{y}} & 0 \\
0 & \frac{\partial \Delta i_{y}}{\partial y} & 0 & 0 & \frac{\partial \Delta i_{y}}{\partial v_{y}} & 0 \\
\frac{\partial \Delta M^{\prime}}{\partial x} & 0 & \frac{\partial \Delta M^{\prime}}{\partial z} & \frac{\partial \Delta M^{\prime}}{\partial v_{x}} & 0 & \frac{\partial \Delta M^{\prime}}{\partial v_{z}}
\end{array}\right]
$$

Then the following equations can be obtained

$$
\boldsymbol{M}^{-1}=\left[\frac{\partial\left(D, \Delta e_{x}, \Delta e_{y}, \Delta i_{x}, \Delta i_{y}, \Delta M^{\prime}\right)}{\partial\left(x, y, z, v_{x}, v_{y}, v_{z}\right)}\right]=\boldsymbol{\Phi}\left[\frac{\partial\left(x, y, z, v_{x}, v_{y}, v_{z}\right)}{\partial(\Delta a, \Delta e, \Delta i, \Delta \Omega, \Delta \omega, \Delta M)}\right]^{-1}=\boldsymbol{\Phi} \boldsymbol{B}^{-1}(t)
$$

\subsection{Solving $B^{-1}(t)$}

Let $\boldsymbol{B}(t)=\left[\frac{\partial\left(x, y, z, v_{x}, v_{y}, v_{z}\right)}{\partial(\Delta a, \Delta e, \Delta i, \Delta \Omega, \Delta \omega, \Delta M)}\right]_{t}, \eta=\sqrt{1-e^{2}}, s_{\alpha}=\sin \alpha, \quad c_{\alpha}=\cos \alpha, \alpha$ is an angle chosen at will and $\boldsymbol{X}(t)=\left[\begin{array}{lllllll}x & y & z & v_{x} & v_{y} & v_{z}\end{array}\right]^{\mathrm{T}}$. Lane and Axelrad [11] has given the expressions of $\boldsymbol{B}(t)$ as shown in Equation (9). The relative state vector $\boldsymbol{X}(t)$ can be changed to $\boldsymbol{X}(t)^{\prime}$, which is obtained by coordinate transformation without considering correlative velocity. Afterwards, let $\mathbf{B}(t)^{\prime}=\frac{\partial\left(\boldsymbol{X}^{\prime}(t)\right)^{\mathrm{T}}}{\partial \Delta \mathbf{O E}^{\mathrm{T}}}$, which can be expressed in Equation (10).

Combining Equation (9) and Equation (10), the equations shown in Equation (11) can be obtained.

$$
\begin{aligned}
& \boldsymbol{B}(t)=\left[\begin{array}{cccccc}
-\frac{3 a n\left(t-t_{0}\right) \eta}{2 r} & a\left(1+\frac{1}{\eta^{2}} \frac{r}{a}\right) \sin \theta & 0 & r \cos i & r & \frac{a^{2} \eta}{r} \\
0 & 0 & -r \sin u & r \sin \cos u & 0 & 0 \\
-\frac{r}{a}+\frac{3 n\left(t-t_{0}\right) e \sin \theta}{2 \eta} & a \cos \theta & 0 & 0 & 0 & -\frac{\text { aesin } \theta}{\eta} \\
\frac{3 a^{2}}{2 r^{2}} n^{2}\left(t-t_{0}\right) e \sin \theta-\frac{3 a}{2 r} \eta \eta & n \eta\left(1+\frac{r}{p}\right)\left(\frac{a^{3}}{r^{2}}\right) \cos \theta+\frac{a e n \sin ^{2} \theta}{\eta^{3}} & 0 & \frac{a e n \operatorname{cosisin} \theta}{\eta} & \frac{\text { aensin } \theta}{\eta}-e n \sin \theta\left(\frac{a^{3}}{r^{2}}\right) \\
0 & 0 & -\frac{a n}{\eta}(\cos u+e \cos \omega)-\frac{a n}{\eta} \sin i(\sin u+e \sin \omega) & 0 & 0 \\
\frac{n a \sin \theta}{\eta} \frac{e}{2 a}+\frac{3 a^{2}}{2 r^{2}} n^{2}\left(t-t_{0}\right) \cos \theta & -n \sin \theta \eta\left(\frac{a^{3}}{r^{2}}\right) & 0 & 0 & 0 & - \text { en } \cos \theta\left(\frac{a^{3}}{r^{2}}\right)
\end{array}\right] \\
& \boldsymbol{B}(t)^{\prime}=\left[\begin{array}{cccccc}
0 & a\left(1+\frac{1}{\eta^{2}} \frac{r}{a}\right) \sin \theta & 0 & r \operatorname{cosi} & r & \frac{a^{2} \eta}{r} \\
0 & 0 & -r \sin u & r \operatorname{sinicos} u & 0 & 0 \\
-\frac{r}{a} & a \cos \theta & 0 & 0 & 0 & -\frac{a e s i n}{\eta} \\
-\frac{n a}{2 r} \eta & \frac{n a}{\eta} \frac{e+\cos \theta}{\eta^{2}} & 0 & \frac{a e n \operatorname{cosisin} \theta}{\eta} & \frac{a e n \sin \theta}{\eta} & 0 \\
0 & 0 & -\frac{a n}{\eta}(\cos u+e \cos \omega) & -\frac{a n}{\eta} \sin i(\sin u+e \sin \omega) & 0 & 0 \\
\frac{n a \sin \theta}{\eta} \frac{e}{2 a} & \frac{n a \sin \theta}{\eta} \frac{a}{r} & 0 & \frac{n a^{2} \eta}{r} \operatorname{cosi} & \frac{n a^{2} \eta}{r} & n\left(\frac{a^{3}}{r^{2}}\right)
\end{array}\right]
\end{aligned}
$$




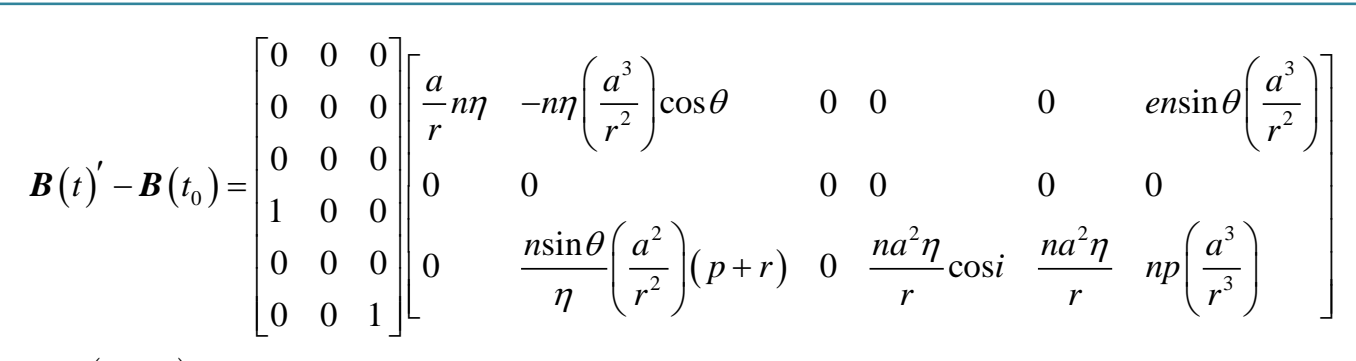

where $p=a\left(1-e^{2}\right)$.

Poisson brackets of orbital elements are well-known, which can be expressed as followings

$$
\begin{aligned}
& \{a, M\}=-\frac{2}{n a} \quad\{e, M\}=-\frac{1-e^{2}}{n a^{2} e} \quad\{i, \omega\}=-\frac{1}{n a^{2} \sqrt{1-e^{2}}} \frac{\cos i}{\sin i} \\
& \{e, \omega\}=\frac{\sqrt{1-e^{2}}}{n a^{2} e} \quad\{i, \Omega\}=\frac{1}{n a^{2} \sqrt{1-e^{2}}} \frac{1}{\sin i}
\end{aligned}
$$

Then the Poisson Matrix can be written as

$$
\boldsymbol{P}=\left[\begin{array}{cccccc}
0 & 0 & 0 & 0 & 0 & -\frac{2}{n a} \\
0 & 0 & 0 & 0 & \frac{\eta}{n a^{2} e} & -\frac{\eta^{2}}{n a^{2} e} \\
0 & 0 & 0 & \frac{1}{\eta n a^{2}} \frac{1}{\sin i} & -\frac{1}{\eta n a^{2}} \frac{\operatorname{cosi}}{\operatorname{sini}} & 0 \\
0 & 0 & -\frac{1}{\eta n a^{2}} \frac{1}{\sin i} & 0 & 0 & 0 \\
0 & -\frac{\eta}{n a^{2} e} & \frac{1}{\eta n a^{2}} \frac{\operatorname{cosi}}{\sin i} & 0 & 0 & 0 \\
\frac{2}{n a} & \frac{\eta^{2}}{n a^{2} e} & 0 & 0 & 0 & 0
\end{array}\right]
$$

Let $\boldsymbol{S}=\left[\begin{array}{cc}\boldsymbol{0} & \boldsymbol{I}_{3} \\ -\boldsymbol{I}_{3} & \boldsymbol{0}\end{array}\right]$, according to $\boldsymbol{B}^{\prime-1}=\boldsymbol{P} \boldsymbol{B}^{\prime T} \boldsymbol{S}^{-1}$, the expression of $\boldsymbol{B}^{\prime-1}$ can be obtained as

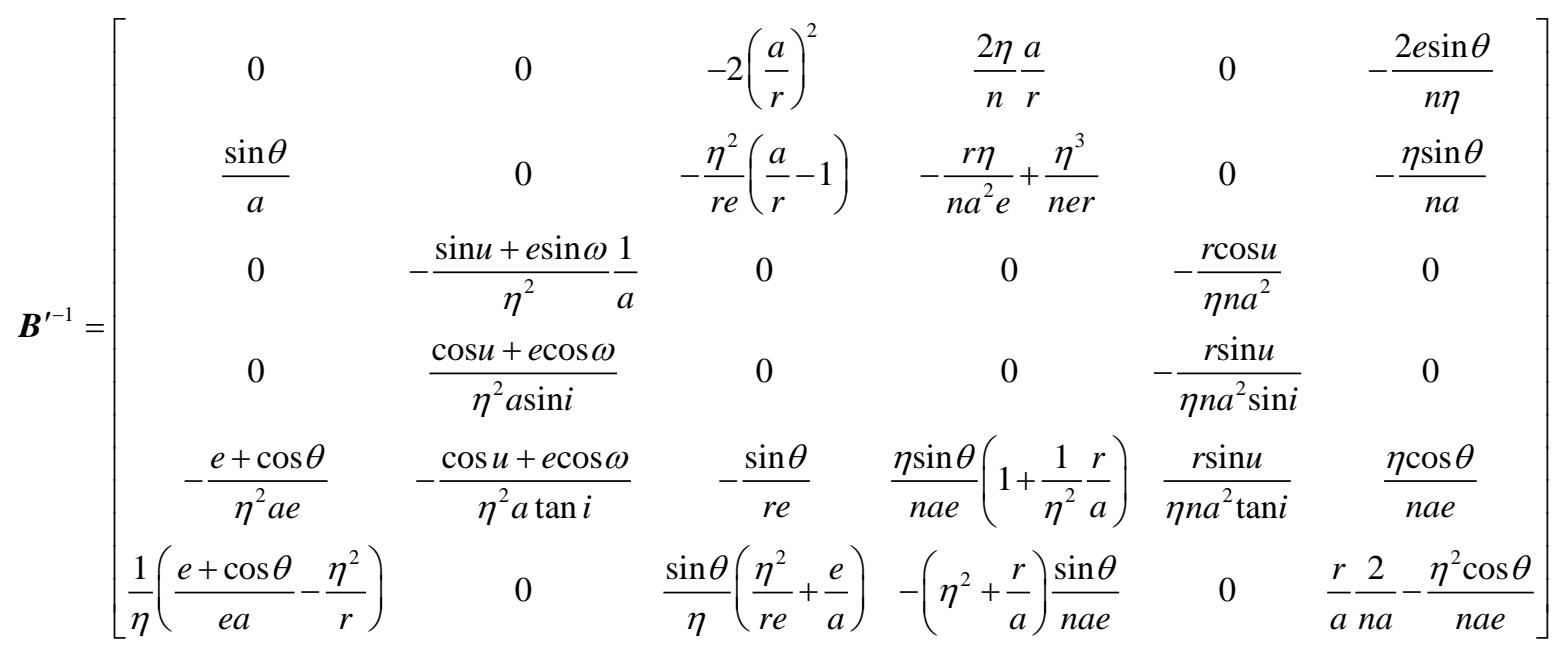

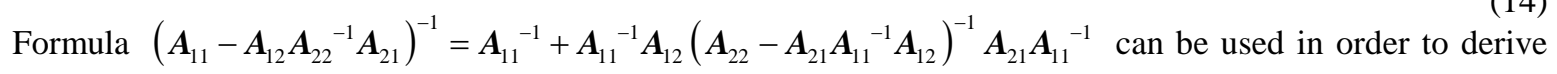


$\boldsymbol{B}^{-1}\left(t_{0}\right)$. Let

$$
\begin{aligned}
& \boldsymbol{A}_{11}=\boldsymbol{B}(t)^{\prime}, \quad \boldsymbol{A}_{12}=\left[\begin{array}{llllll}
0 & 0 & 0 & 0 & 0 & 1 \\
0 & 0 & 0 & 0 & 0 & 0 \\
0 & 0 & 0 & 1 & 0 & 0
\end{array}\right]^{\mathrm{T}}, \quad \boldsymbol{A}_{22}=\left[\begin{array}{lll}
1 & 0 & 0 \\
0 & 1 & 0 \\
0 & 0 & 1
\end{array}\right] \\
& \boldsymbol{A}_{21}=\left[\begin{array}{cccccc}
\frac{a}{r} n \eta & -n \eta\left(\frac{a^{3}}{r^{2}}\right) \cos \theta & 0 & 0 & 0 & \text { ensin } \theta\left(\frac{a^{3}}{r^{2}}\right) \\
0 & 0 & 0 & 0 & 0 & 0 \\
0 & \frac{n \sin \theta}{\eta}\left(\frac{a^{2}}{r^{2}}\right)(p+r) & 0 & \frac{n a^{2} \eta}{r} \operatorname{cosi} & \frac{n a^{2} \eta}{r} & n p\left(\frac{a^{3}}{r^{3}}\right)
\end{array}\right]
\end{aligned}
$$

Afterwards, $\boldsymbol{B}\left(t_{0}\right)=\boldsymbol{A}_{11}-\boldsymbol{A}_{12} \boldsymbol{A}_{22}^{-1} \boldsymbol{A}_{21}$, hence $\boldsymbol{B}^{-1}(t)$ can be expressed as

$$
\boldsymbol{B}^{-1}(t)=\left[\begin{array}{cccccc}
1 & 0 & 0 & 0 & 0 & 0 \\
0 & 1 & 0 & 0 & 0 & 0 \\
0 & 0 & 1 & 0 & 0 & 0 \\
0 & 0 & 0 & 1 & 0 & 0 \\
0 & 0 & 0 & 0 & 1 & 0 \\
-\frac{3 n\left(t-t_{0}\right)}{2 a} & 0 & 0 & 0 & 0 & 1
\end{array}\right]^{-1} \boldsymbol{B}^{-1}\left(t_{0}\right)
$$

\subsection{Solving $M^{-1}$}

According to Equation (2), Equation (8) and Equation (12), the expressions of $\boldsymbol{M}^{-1}$ can be easily obtained. This method leads to very simple results, and easy to simplify. $\boldsymbol{M}^{-1}$ can be expressed in two ways: including $\dot{r}$ and without $\dot{r}$. The detailed results are given in Appendix A.

Obviously the in-plane and out-of-plane motions can be decoupled based on the relative orbit elements. Using $\boldsymbol{M}$ and $\boldsymbol{M}^{-1}$, the initial relative position and relative velocity can be used to describe the relative trajectory too.

$$
\boldsymbol{X}(t)=\boldsymbol{M}(t) \boldsymbol{M}^{-1}\left(t_{0}\right) \boldsymbol{X}\left(t_{0}\right)
$$

Equation (16) is equivalent to Lawden's equations but with a much higher accuracy. Meanwhile the introduction of the integrals as in Lawden's equations is avoided. The finite difference form can be obtained to design control strategy as follow

$$
\left[\begin{array}{c}
\mathrm{d} D \\
\mathrm{~d} \Delta e_{x} \\
\mathrm{~d} \Delta e_{y} \\
\mathrm{~d} \Delta i_{x} \\
\mathrm{~d} \Delta i_{y} \\
\mathrm{~d} \Delta M^{\prime}
\end{array}\right]=\left[\begin{array}{ccc}
\frac{\partial D}{\partial \Delta v_{x}} & 0 & \frac{\partial D}{\partial \Delta v_{z}} \\
\frac{\partial \Delta e_{x}}{\partial \Delta v_{x}} & 0 & \frac{\partial \Delta e_{x}}{\partial \Delta v_{z}} \\
\frac{\partial \Delta e_{y}}{\partial \Delta v_{x}} & 0 & \frac{\partial \Delta e_{y}}{\partial \Delta v_{z}} \\
0 & \frac{\partial \Delta i_{x}}{\partial \Delta v_{y}} & 0 \\
0 & \frac{\partial \Delta i_{y}}{\partial \Delta v_{y}} & 0 \\
\frac{\partial \Delta M^{\prime}}{\partial \Delta v_{y}} & 0 & \frac{\partial \Delta M^{\prime}}{\partial \Delta v_{z}}
\end{array}\right]\left[\begin{array}{c}
\Delta v_{x} \\
\Delta v_{y} \\
\Delta v_{z}
\end{array}\right]
$$


Take $\Delta v$ as perturbation acceleration $f$, the equations above would become differential equations. Based on the differential equations and the finite difference form, the perturbation effects and control problem can be easily solved.

\section{Numerical Simulations}

In this section, a numerical simulation is proposed to demonstrate the performance of the ROEs-based relative motion equations. Suppose that at the beginning the chief and deputy satellites have the same location and velocity in space. The two satellites move on an elliptical orbit with semi-major axis $7555 \mathrm{~km}$ and inclination of angle $48^{\circ}$. The initial right ascension of ascending node, argument of perigee, and true anomaly of the satellites are all set to be zero. Let $e_{0}=0.3$. Let $\Delta d=1000 \mathrm{~m}$. The desired relative orbit elements are

$$
\left(\begin{array}{llllll}
D & \Delta e_{x} & \Delta e_{y} & \Delta i_{x} & \Delta i_{y} & \Delta M^{\prime}
\end{array}\right)=\left(\begin{array}{llllll}
0 & \Delta d / a & \Delta d / a & 2 \Delta d / a & 2 \Delta d / a & 0
\end{array}\right)
$$

The relative orbit trajectory for deputy satellite with respect to the chief established with the ROE-based control law is shown in the chief satellite $\boldsymbol{F}_{o}$ frame in Figure 2. The chief and the deputy satellites are assumed to be coincident initially. Thus the relative orbit starts from the origin. The $\diamond$ in Figure 2 denotes the thrust location. The control laws can be obtained from the inverse matrix of the relative state transition matrix (Table 1).

Table 1. The control strategy of formation establishment.

\begin{tabular}{ccc}
\hline Sequence & Thrust location $(\theta)$ & Impulsive velocity in $\boldsymbol{F}_{o}$ frame $(\mathrm{m} / \mathrm{s})$ \\
\hline 1 & 0.9992 & {$[0.1452,0,0.0592]$} \\
2 & 3.7132 & {$[-0.4238,0,0.092]$} \\
3 & 5.4978 & {$[0,3.4553,0]$} \\
4 & 7.2824 & {$[0.1274,0,0]$} \\
\hline
\end{tabular}
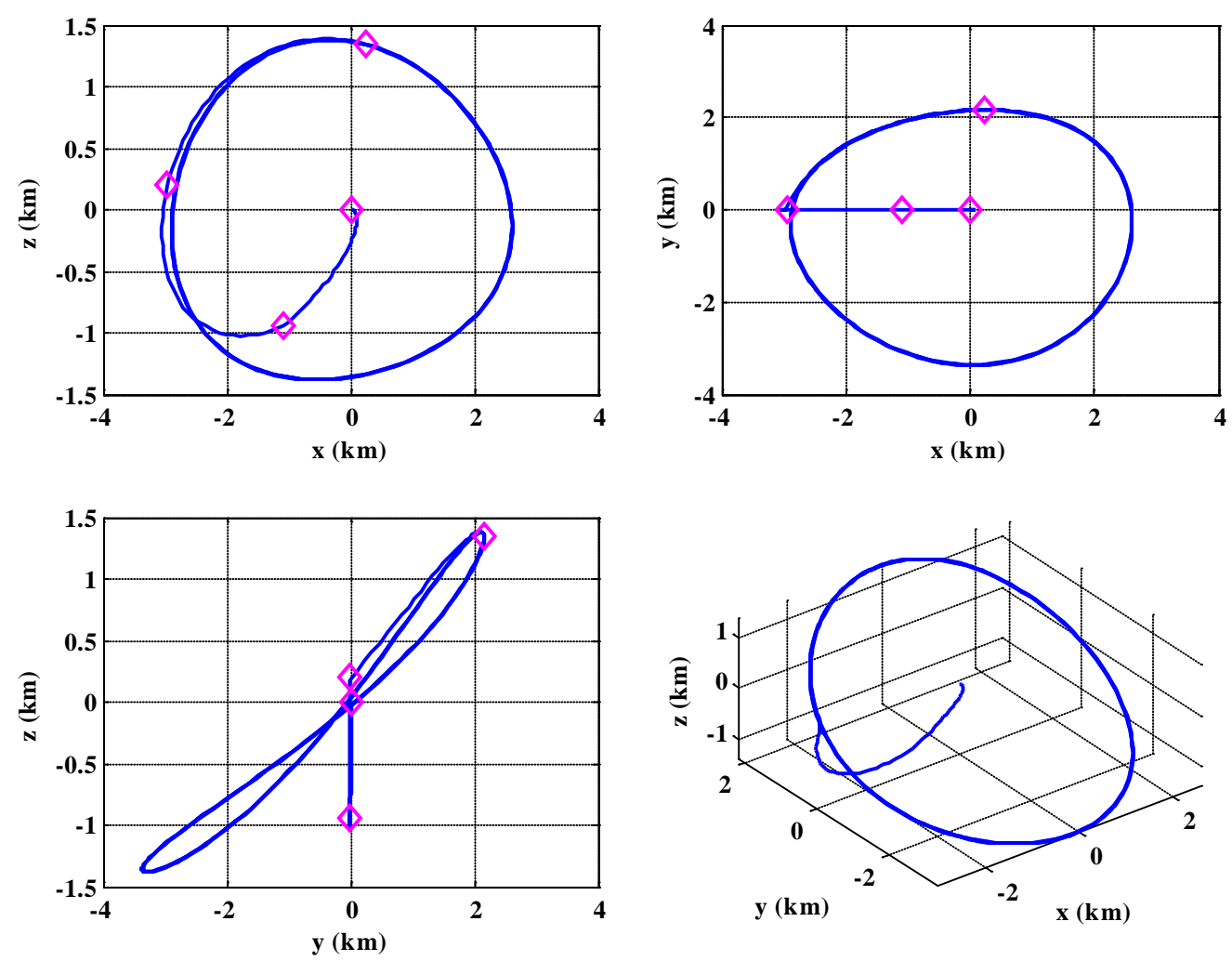

Figure 2. Relative orbit trajectory for formation establishment. 


\section{Conclusion}

The paper investigated the problem of the inverse transformation of the state transition matrix based on relative orbit elements. Poisson bracket theory is used to obtain the linear transformations between the two representations: the relative orbit elements and the geocentric orbital frame. This new method leads to very simple results, and very easy to simplify. Two different expressions of the inverse matrix are presented, which can be used to analyze the elliptical relative motion control strategy and perturbation effects.

\section{References}

[1] Casotto, S. (2010) Position and Velocity Perturbations in the Orbital Frame in Terms of Classical Element Perturbations. Celestial Mechanics and Dynamical Astronomy, 55, 209-221. http://dx.doi.org/10.1007/BF00692510

[2] Garrison, J.L., Gardner, J.L. and Axelrad, P. (1995) Relative Motion in Highly Elliptical Orbits. Paper No. AAS 95-194, Space Flight Mechanics Conference, Alburquerque.

[3] Alfriend, K.T., Schaub, H. and Gim, D.-W. (2000) Gravitational Perturbations, Nonlinearity and Circular Orbit Assumption Effects on Formation Flying Control Strategies. AAS Guidance and Control Conference, Breckenridge, CO, AAS 00-012, 139-158.

[4] Gim, D.-W. and Alfriend, K.T. (2005) Satellite Relative Motion Using Differential Equinoctial Elements. Celestial Mechanics and Dynamical Astronomy, 92, 295-336. http://dx.doi.org/10.1007/s10569-004-1799-0

[5] Sengupta, P., Vadali, S.R. and Alfriend, K.T. (2006) Second-Order State Transition for Relativemotion near Perturbed, Elliptic Orbits. Celestial Mechanics and Dynamical Astronomy, 97, 101-129. http://dx.doi.org/10.1007/s10569-006-9054-5

[6] Fontdecaba, J. (2008) Dynamics of Formation Flying Applications to Earth and Universe Observation. Observatoire de la Côte d'Azur, Grasse.

[7] Chobotov, V. (2002) Orbital Mechanics. American Institute of Aeronautics and Astronautics, Reston.

[8] Han, C. and Yin, J. (2011) Satellite Relative Motion in Elliptical Orbit Using Relative Orbit Elements. American Astronautical Society, Paper AAS 11-497.

[9] Han, C. and Yin, J. (2012) Formation Design in Elliptical Orbit Using Relative Orbit Elements. Acta Astronautica, 77, 34-47. http://dx.doi.org/10.1016/j.actaastro.2012.02.026

[10] Yin, J. and Han, C. (2012) Perturbation Effects on Elliptical Relative Motion Based on Relative Orbit Elements. American Astronautical Society, Paper AAS 12-148.

[11] Lane, C. and Axelrad, P. (2006) Formation Design in Eccentric Orbits Using Linearized Equations of Relative Motion. Journal of Guidance, Control, and Dynamics, 29, 146-160. http://dx.doi.org/10.2514/1.13173 


\section{Appendix A}

1) Including $\dot{r}$

$$
\begin{aligned}
& \frac{\partial D}{\partial x}=\frac{3 \dot{r} \sqrt{1-e^{2}}}{a^{2}}\left(\frac{a}{r}\right)^{2} \quad \frac{\partial D}{\partial z}=\frac{3 n(2+e \cos \theta)}{a}\left(\frac{a}{r}\right)^{2} \quad \frac{\partial D}{\partial v_{x}}=-\frac{3}{r} \sqrt{1-e^{2}} \quad \frac{\partial D}{\partial v_{z}}=\frac{3 \dot{r}}{n a^{2}} \\
& \frac{\partial \Delta M^{\prime}}{\partial x}=\frac{1}{r}-\frac{\dot{r} \sqrt{1-e^{2}}}{n a} \bar{\eta}\left(1+\frac{1}{1-e^{2}} \frac{r}{a}\right) \frac{\sin \theta}{r}-\frac{3 \dot{r} \sqrt{1-e^{2}}}{a^{2}}\left(\frac{a}{r}\right)^{2}\left(t-t_{0}\right) \\
& \frac{\partial \Delta M^{\prime}}{\partial z}=-\frac{\left[a\left(1-e^{2}\right)+2 r\right]}{r^{2}} \bar{\eta} \sin \theta+\frac{e \sin \theta}{a \sqrt{1-e^{2}}}-\frac{3 n(2+e \cos \theta)}{a}\left(\frac{a}{r}\right)^{2}\left(t-t_{0}\right) \\
& \frac{\partial \Delta M^{\prime}}{\partial v_{x}}=\left(1+\frac{1}{1-e^{2}} \frac{r}{a}\right) \sqrt{1-e^{2}} \bar{\eta} \frac{\sin \theta}{n a}+\frac{3 \sqrt{1-e^{2}}\left(t-t_{0}\right)}{r} \quad \frac{\partial \Delta M^{\prime}}{\partial v_{z}}=\frac{\sqrt{1-e^{2}}}{n a} \bar{\eta} \cos \theta+\frac{r}{a} \frac{2}{n a}-\frac{3 \dot{r}}{n a^{2}}\left(t-t_{0}\right) \\
& \frac{\partial \Delta e_{x}}{\partial x}=-\frac{\dot{r} \sqrt{1-e^{2}}}{n a r} \cos u+\left(\frac{1}{a}-\frac{1}{r}\right) \sin u \frac{\partial \Delta e_{x}}{\partial z}=-3\left(1-e^{2}\right) \frac{a}{r} \frac{1}{r} \cos u-\frac{\dot{r} \sqrt{1-e^{2}}}{n a} \frac{2}{r} \sin u \\
& \frac{\partial \Delta e_{x}}{\partial v_{x}}=\frac{2 \sqrt{1-e^{2}}}{n a} \cos u+\frac{\dot{r}}{n^{2} a^{2}} \frac{r}{a} \sin u \quad \frac{\partial \Delta e_{x}}{\partial v_{z}}=-\frac{\sqrt{1-e^{2}}}{n a} \sin u \quad \frac{\partial \Delta e_{y}}{\partial x}=-\frac{\dot{r} \sqrt{1-e^{2}}}{n a r} \sin u+\left(\frac{1}{r}-\frac{1}{a}\right) \cos u \\
& \frac{\partial \Delta e_{y}}{\partial z}=-3\left(1-e^{2}\right)\left(\frac{a}{r}\right) \frac{1}{r} \sin u+\frac{\dot{r} \sqrt{1-e^{2}}}{n a} \frac{2}{r} \cos u \quad \frac{\partial \Delta e_{y}}{\partial v_{x}}=\frac{2 \sqrt{1-e^{2}}}{n a} \sin u-\frac{\dot{r}}{n^{2} a^{2}} \frac{r}{a} \cos u \\
& \frac{\partial \Delta e_{y}}{\partial v_{z}}=\frac{\sqrt{1-e^{2}}}{n a} \cos u \quad \frac{\partial \Delta i_{x}}{\partial y}=\frac{\cos u}{r}+\frac{\dot{r} \sin u}{\sqrt{1-e^{2}} n a^{2}} \quad \frac{\partial \Delta i_{x}}{\partial v_{y}}=-\frac{r \sin u}{\sqrt{1-e^{2}} n a^{2}} \quad \frac{\partial \Delta i_{y}}{\partial y}=\frac{\sin u}{r}-\frac{\dot{r} \cos u}{\sqrt{1-e^{2}} n a^{2}} \\
& \frac{\partial \Delta i_{y}}{\partial v_{y}}=\frac{r \cos u}{\sqrt{1-e^{2}} n a^{2}} \quad \text { where } \dot{r}=\frac{n a e \sin \theta}{\sqrt{1-e^{2}}} \quad \bar{\eta}=\frac{1-\sqrt{1-e^{2}}}{e} \text {. }
\end{aligned}
$$

2) Without $\dot{r}$

$$
\begin{aligned}
& \frac{\partial D}{\partial x}=\frac{3 n e \sin \theta}{a}\left(\frac{a}{r}\right)^{2} \quad \frac{\partial D}{\partial z}=\frac{3 n(2+e \cos \theta)}{a}\left(\frac{a}{r}\right)^{2} \quad \frac{\partial D}{\partial v_{x}}=-\frac{3}{r} \sqrt{1-e^{2}} \quad \frac{\partial D}{\partial v_{z}}=\frac{3 e \sin \theta}{a \eta} \\
& \frac{\partial \Delta M^{\prime}}{\partial x}=\frac{1}{r}-\left(1-\sqrt{1-e^{2}}\right) \frac{\sin ^{2} \theta}{r}\left(1+\frac{1}{1-e^{2}} \frac{r}{a}\right)-\frac{3 n e \sin \theta}{a}\left(\frac{a}{r}\right)^{2}\left(t-t_{0}\right) \\
& \frac{\partial \Delta M^{\prime}}{\partial z}=-\frac{\left[a\left(1-e^{2}\right)+2 r\right]}{r^{2}} \bar{\eta} \sin \theta+\frac{e \sin \theta}{a \sqrt{1-e^{2}}}-\frac{3 n(2+e \cos \theta)}{a}\left(\frac{a}{r}\right)^{2}\left(t-t_{0}\right) \\
& \frac{\partial \Delta M^{\prime}}{\partial v_{x}}=\left(\frac{1}{\sqrt{1-e^{2}}} \frac{r}{a}+\sqrt{1-e^{2}}\right) \bar{\eta} \frac{\sin \theta}{n a}+\frac{3 \sqrt{1-e^{2}}\left(t-t_{0}\right)}{r} \quad \frac{\partial \Delta M^{\prime}}{\partial v_{z}}=\frac{\sqrt{1-e^{2}}}{n a} \bar{\eta} \cos \theta+\frac{r}{a} \frac{2}{n a}-\frac{3 e \sin \theta}{a \eta}\left(t-t_{0}\right) \\
& \frac{\partial \Delta e_{x}}{\partial x}=-\frac{e \sin \theta}{r} \cos u+\left(\frac{1}{a}-\frac{1}{r}\right) \sin u \quad \frac{\partial \Delta e_{x}}{\partial z}=-3\left(1-e^{2}\right) \frac{a}{r} \frac{1}{r} \cos u-\frac{2}{r} e \sin \theta \sin u \\
& \frac{\partial \Delta e_{x}}{\partial v_{x}}=\frac{2 \sqrt{1-e^{2}}}{n a} \cos u+\frac{e \sin \theta}{n a \eta} \frac{r}{a} \sin u \quad \frac{\partial \Delta e_{x}}{\partial v_{z}}=-\frac{\sqrt{1-e^{2}}}{n a} \sin u \quad \frac{\partial \Delta e_{y}}{\partial x}=-\frac{e \sin \theta}{r} \sin u+\left(\frac{1}{r}-\frac{1}{a}\right) \cos u \\
& \frac{\partial \Delta e_{y}}{\partial z}=-3\left(1-e^{2}\right)\left(\frac{a}{r}\right) \frac{1}{r} \sin u+\frac{2}{r} e \sin \theta \cos u \quad \frac{\partial \Delta e_{y}}{\partial v_{x}}=\frac{2 \sqrt{1-e^{2}}}{n a} \sin u-\frac{e \sin \theta}{n a \eta} \frac{r}{a} \cos u \\
& \frac{\partial \Delta e_{y}}{\partial v_{z}}=\frac{\sqrt{1-e^{2}}}{n a} \cos u \quad \frac{\partial \Delta i_{x}}{\partial y}=\frac{\cos u}{r}+\frac{e \sin \theta \sin u}{\left(1-e^{2}\right) a} \quad \frac{\partial \Delta i_{x}}{\partial v_{y}}=-\frac{r \sin u}{\sqrt{1-e^{2}} n a^{2}} \frac{\partial \Delta i_{y}}{\partial y}=\frac{\sin u}{r}-\frac{e \sin \theta \cos u}{\left(1-e^{2}\right) a} \\
& \frac{\partial \Delta i_{y}}{\partial v_{y}}=\frac{r \cos u}{\sqrt{1-e^{2}} n a^{2}} .
\end{aligned}
$$


Scientific Research Publishing (SCIRP) is one of the largest Open Access journal publishers. It is currently publishing more than 200 open access, online, peer-reviewed journals covering a wide range of academic disciplines. SCIRP serves the worldwide academic communities and contributes to the progress and application of science with its publication.

Other selected journals from SCIRP are listed as below. Submit your manuscript to us via either submit@scirp.org or Online Submission Portal.
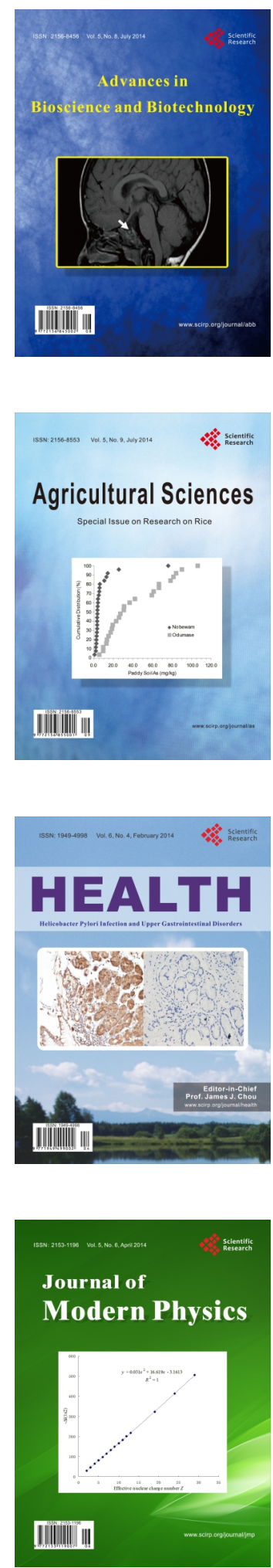
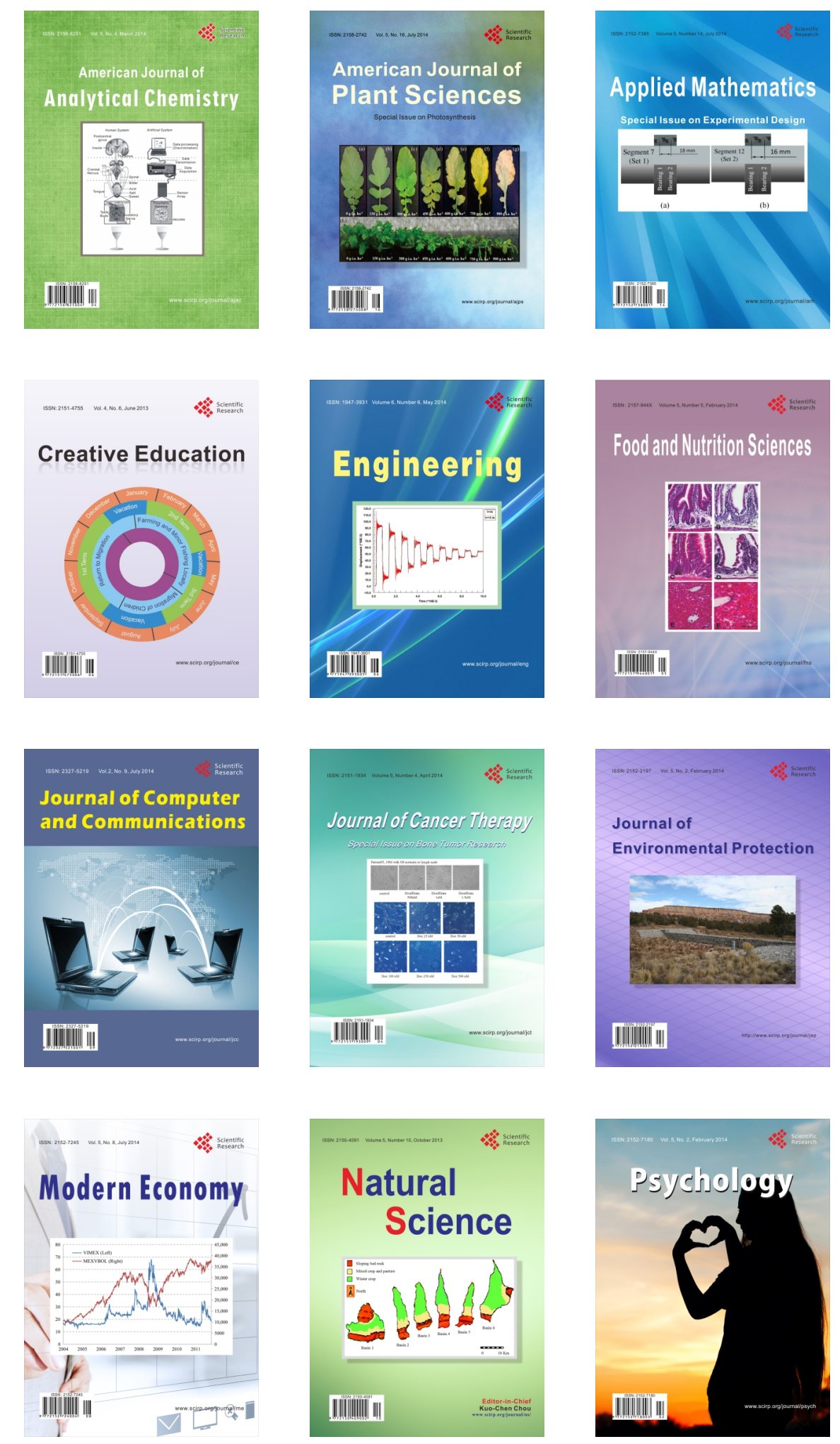